\title{
TEST OF THE GEM FRONT TRACKER FOR THE SBS SPECTROMETER AT JEFFERSON LAB
}

\author{
F. Mammoliti ${ }^{1,2, a}$, V. Bellini ${ }^{1,2}$, E. Cisbani ${ }^{3,4}$, F. Librizzi ${ }^{2}$, P. Musico ${ }^{5}$, F. Noto ${ }^{1,2}$, R. Perrino ${ }^{6}$, and M.C. Sutera ${ }^{1,2}$ \\ ${ }^{1}$ Dipartimento di Fisica ed Astronomia, Università di Catania, via Santa Sofia 64, I-95123 Catania, Italy \\ ${ }^{2}$ INFN - Sezione di Catania, via Santa Sofia 64, I-95123 Catania, Italy \\ ${ }^{3}$ INFN - Sezione di Roma La Sapienza, P.le Aldo Moro, 2 I-00185 Roma, Italy \\ ${ }^{4}$ Istituto Superiore di Sanità, viale Regina Elena 299, I-00161 Roma, Italy \\ ${ }^{5}$ INFN - Sezione di Genova, via Dodecaneso 33, I-16146 Genova, Italy \\ ${ }^{6}$ INFN - Sezione di Lecce, via Arnesano, I-73100 Lecce, Italy
}

\begin{abstract}
A new Large-Acceptance Forward Angle Spectrometer (Super BigBite) is under development at JLab/Hall A to optimally exploit the exciting opportunities offered by the $12 \mathrm{GeV}$ upgrade of the electron beam. The tracker of this new apparatus is based on the Gas Electron Multiplier (GEM) technology, which has been chosen to optimize cost/performance, position resolution and to meet the high hit rate $\left(>1 \mathrm{MHz} / \mathrm{cm}^{2}\right)$. The first GEM detector modules, designed and built by the INFN Collaboration JLAB12, were tested at the DESY test beam facility in Hamburg, by using an electron beam with energy ranging from 2.0 to $6.0 \mathrm{GeV}$. In particular, two 40x50 $\mathrm{cm}^{2}$ and one $10 \times 10 \mathrm{~cm}^{2}$ GEM chambers were equipped with a new implementation of the APV25 readout chip. Measurements were performed at different impact points and angles between the electron beam and the plane of the GEM chambers, with one large chamber in a solenoid magnetic field up to 500 Gauss. In this report we present the technical characteristics of the detector and comment on the presently achieved performance.
\end{abstract}

\section{Introduction}

The Jefferson Laboratory (JLab) [1] is one of the most important experimental facility providing a multi $\mathrm{GeV}$, high intensity, longitudinally polarized, electron beam. The laboratory is undergoing a major upgrade of its CEBAF electron beam and experimental halls. In late 2014, CEBAF will deliver electron with energy up to $12 \mathrm{GeV}$ (twice the previous limit) with excellent intensity (up to $100 \mu A$ ) and longitudinal polarization (up to $85 \%$ ). In order to take advantage of the new scenario, the equipments of the 3 existing experimental Halls are under upgrading to optimally exploit the opportunities of the new beam. In particular members of Hall A collaboration are developing a new reconfigurable spectrometer, the Super BigBite (SBS [2], fig.1), featuring very forward angle (down to 7 degrees), large momentum (2-10 GeV/c) and angular acceptance (64 $\mathrm{mrad})$, high rate capability $\left(1 \mathrm{MHz} / \mathrm{cm}^{2}\right)$ and very high luminosity environment (up to $1039 /\left(\mathrm{s} \cdot \mathrm{cm}^{2}\right)$ ). The new spectrometer will consist, in its full configuration, of a dipole magnet with field integral up to $3 T$ (it will operate at about $2 T$ ), a primary charged particle tracker (first tracker), 2 identical proton polarimeters (made of a Carbon analyzer and large tracker), and an hadron calorimeter. SBS will initially serve 4 experiments [3] dedicated to the study of the nucleon structure in terms of elastic elec-

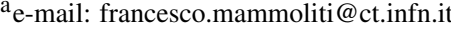

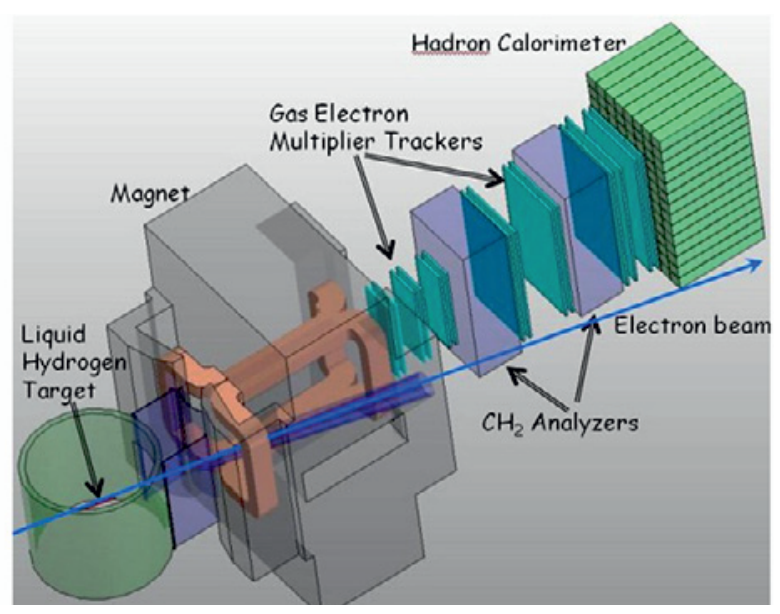

Figure 1. Schematic layout of the SBS Spectrometer: there are three stations with trackers.

tromagnetic form factors at high 4-momentum transfer $Q^{2}$ up to $15 \mathrm{GeV}^{2}$ and of transverse momentum distributions of the quarks in the SIDIS (Semi Inclusive Deep Inelastic Scattering) region. The tracking systems of SBS will be mainly based on GEM chambers. In the next section the main features of the SBS tracker and of the GEM de- 


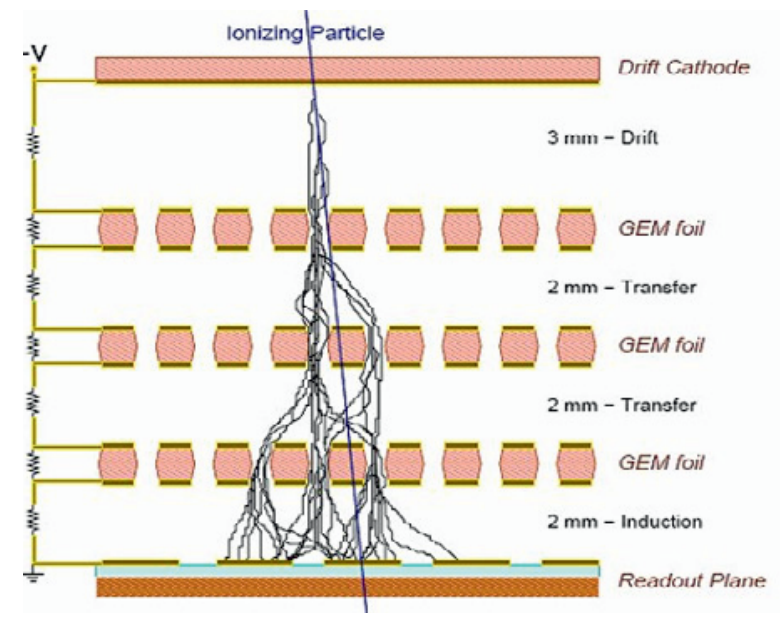

Figure 2. Schematic layout of the GEM Chamber. The total gain is about $10^{6}$.

tector will be presented and finally the results of the test performed at DESY will be discussed.

\section{SBS Tracker and GEM Detector}

The SBS tracking system is made of three stations. The primary (front) tracker, placed just after the dipole momentum analyzing magnet, will consist of six large area $\left(40 \times 150 \mathrm{~cm}^{2}\right)$ and high resolution $(\approx 70 \mu \mathrm{m})$ GEM chambers, for a total tracker length of about $50 \mathrm{~cm}$. Each chamber is made by 3 adjacent GEM modules of 40x50 $\mathrm{cm}^{2}$ active rectangular area, for a total of 18 modules. It is designed to be capable to track accurately particles emerging from the electron scattering in a large background of soft photons $\left(\approx 0.5 \mathrm{MHz} / \mathrm{cm}^{2}\right)$ and MIPs $\left(\approx 0.2 \mathrm{MHz} / \mathrm{cm}^{2}\right)$. The primary tracking will be reinforced by combination with two small $\left(10 \times 20 \mathrm{~mm}^{2}\right)$ planes of silicon $\mu$ strips placed immediately after the target. The other stations are meant to track particles after a polarization analyzer wall and will require less accuracy. The primary tracker is under the responsibility of INFN groups. GEM technology [4] has been chosen to optimize cost/performance, position resolution and meet the high rate $\left(>1 \mathrm{MHz} / \mathrm{cm}^{2}\right)[5]$. The single module is made of 3 GEM foils and double layer $x / y$ strips readout with $400 \mu$ strip pitch (figure 2). The $8 \mathrm{~mm}$ wide mechanical frame incorporates high voltage feeding protection resistors and gas inlet/outlet holes. The signals from each triple GEM module are read out in two coordinates through COMPASS-like [6] strip conductors planes. The front-end electronics [7] (FE) for the $\approx 100 \mathrm{~K}$ channels of the tracker is based on the APV25 [8] chip, successfully used in the LHC experiment CMS. The APV25 is a serial output analogue ASIC running at $40 \mathrm{MHz}$. The FE cards, each with 128 channels, are placed around the GEM module. Custom backplanes are used to distribute power and control to the FE cards and to collect the analogue outputs.

In figure 3 a fully equipped $40 \times 50 \mathrm{~cm}^{2}$ GEM module setup under test at DESY is shown. The module is equipped with the APV25 electronics and 18 front-end

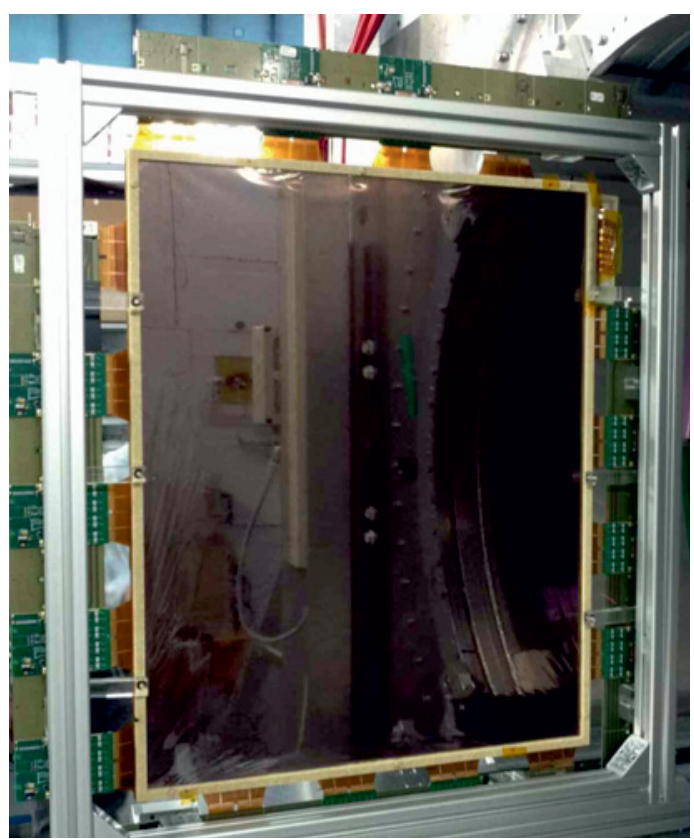

Figure 3. Fully equipped 40x50 $\mathrm{cm}^{2}$ GEM module setup under test at DESY.

cards are located behind the 4 rectangular backplanes that sit along the 4 sides of the module. During the test, a gas mixture of $\mathrm{Ar}(70 \%)$ and $\mathrm{CO}_{2}(30 \%)$ has been used and HV has been powered by the HV-GEM system [9] providing 7 independent HV levels. Moreover, precise tracking has been performed by small scintillators detector located before the GEM. The test was carried out in the T22 DESY Test electron/positron beam area [10]. The test beam is originated from the lepton synchrotron DESY II by converted bremsstrahlung on a carbon fiber target. The energy of the beam varies between about 1 and $6 \mathrm{GeV} / \mathrm{c}$ with typical intensity of 1000 particle/(s. $\left.\mathrm{cm}^{2}\right)$ (divergence is about $2 \mathrm{mrad}$ ).

\section{Data Analysis and Discussion}

In this section we present preliminary results of data analysis performed on about 30 beam runs obtained by using three GEM chambers (one of 10x10 $\mathrm{cm}^{2}$ and two of 40x50 $\mathrm{cm}^{2}$, respectively). All chambers were readout by the APV electronics which were under development at the same time. During the test, different configurations have been used: energy of the electron beam (from 2.0 to $6.0 \mathrm{GeV}$ ), HV settings, angle between the beam and the plane of the chamber and position of the chamber with respect to the beam. Moreover, in order to measure pedestals, without beam runs were acquired. Standard analysis process includes hot channels masking, common noise suppression and pedestal subtraction. A single signal in the $\mathrm{x}$-direction is shown in figure 4 (top panel): it is clearly visible at about strip \#530 and it is obtained after the pedestal subtraction. By using APV 25 chips, it is possible to record different parts of the signal (every $25 \mathrm{~ns}$ ), event by event. 


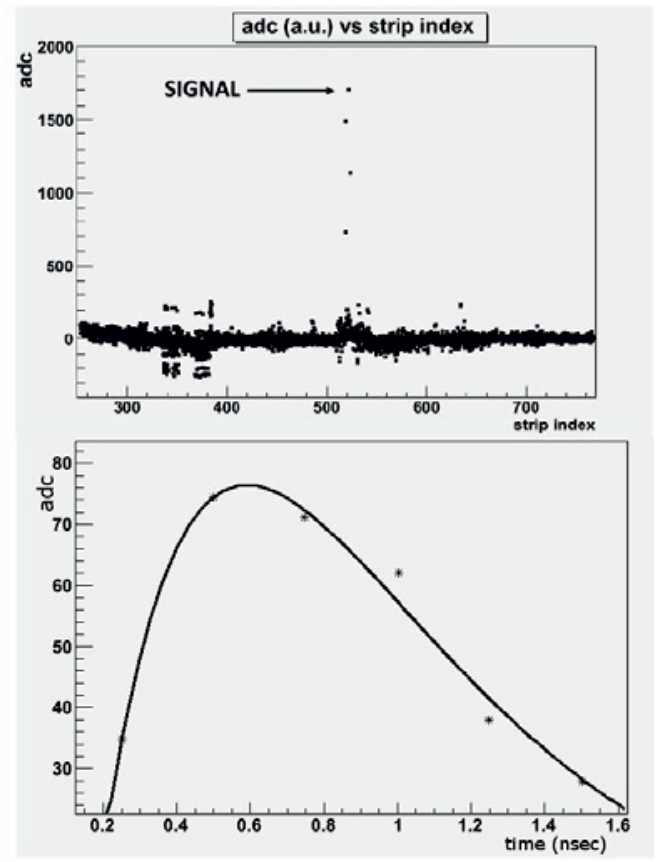

Figure 4. Top panel: single event signal in the $\mathrm{x}$-direction (the value of adc is given in arbitrary units). Bottom panel: signal shape in which the adc's of the single samples are plotted; the continuous line is the fit obtained by the formula (1).

The shape of the signal was fitted by using the formula (1):

$$
A\left[1-\exp \left(-\frac{t-t_{0}}{\tau_{1}}\right)\right] \cdot \exp \left(-\frac{t-t_{0}}{\tau_{2}}\right)
$$

in which $\tau_{1}$ and $\tau_{2}$ are the slope and falling time of the signal, respectively, $t_{0}$ is the start time and $\mathrm{A}$ is the signal amplitude (see figure 4-bottom panel). Adjacent firing strips are grouped in clusters and both number of clusters and number of strips of each cluster was evaluated (figure 5-top and bottom panels, respectively). It is evident that a low number of clusters is present in a single event and moreover there are only few strips in each cluster. In both cases, distributions are consistent with the data from COMPASS GEM characterization [11]. The percentage of events with a minimum of one cluster is about $95 \%$ for all 3 chambers used during the test.

In order to select the single events of a run, we checked if there is a cluster on each chamber in the $\mathrm{x}$-direction (see figure 6). Each cluster provides the hit position $P_{n}\left(Z_{n}, X_{n}\right)$ and its uncertainty $\sigma_{n}$, where $\mathrm{n}$ is the index of the chamber. By using two points, for example $P_{1}\left(Z_{1}, X_{1}\right)$ and $P_{2}\left(Z_{2}, X_{2}\right)$, a straight line $\mathrm{x}=\mathrm{a} \cdot \mathrm{z}+\mathrm{b}$ is reconstructed in the $\mathrm{z}-\mathrm{x}$ twodimensional space ( $\mathrm{a}$ and $\mathrm{b}$ are obtained by a linear fit). Finally, we consider $P_{0}\left(Z_{0}, X_{0}\right)$ and if $\left|X_{0}-\mathrm{a} Z_{0}-\mathrm{b}\right|<\sigma_{0}$ than the signal of the three chambers belongs to the same particle otherwise the event is rejected. The ratio between the number of selected events and the total number of events in which a cluster was found on each chamber gives a percentage value of about $90 \%$. In figure 7-top, the total charge collected in the $\mathrm{y}$ - vs $\mathrm{x}$ - direction is plotted and

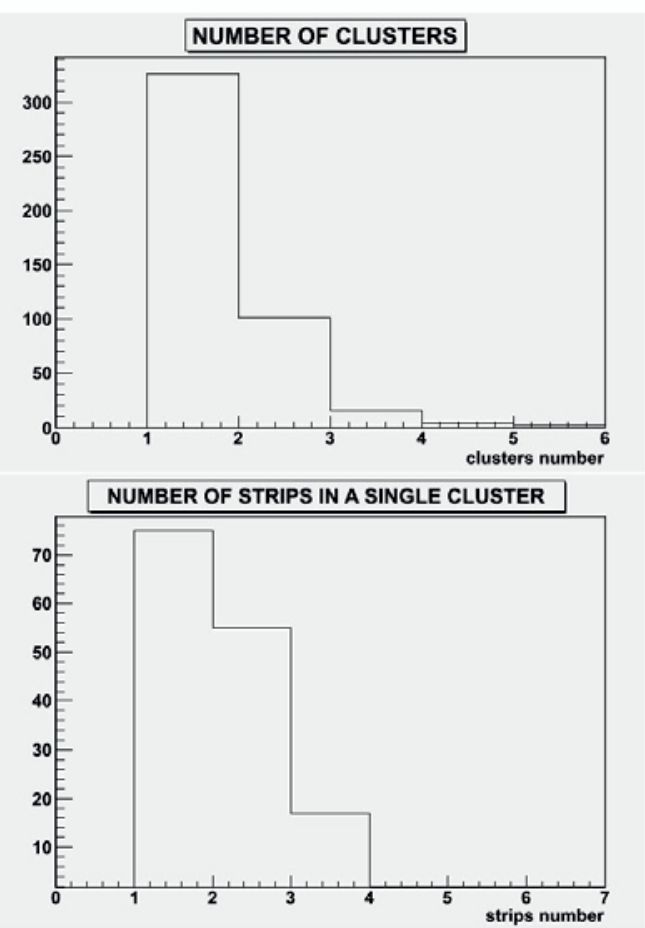

Figure 5. Number of clusters (top panel) and number of strips of a single cluster (bottom panel).

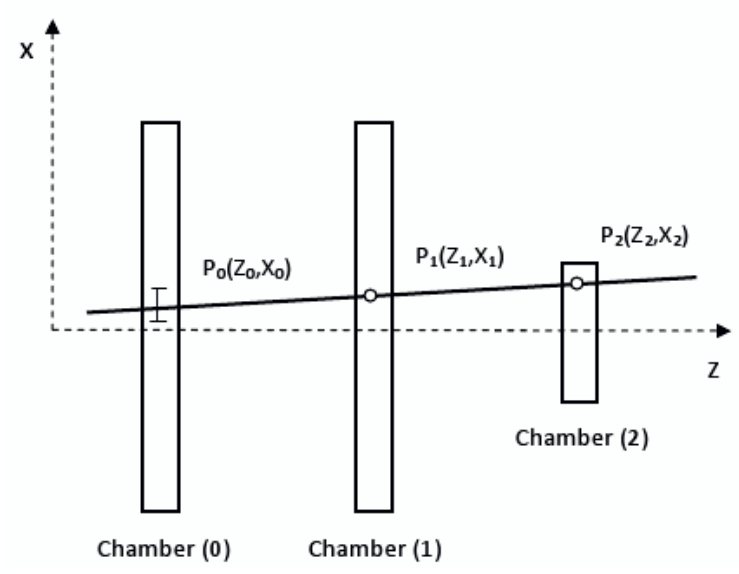

Figure 6. Schematic layout of the three GEM chambers during the test. The active area (perpendicular to the $\mathrm{z}$-axis) has a dimension of $40 \times 50 \mathrm{~cm}^{2}$ for chamber (0) and (1), and of $10 \times 10$ $\mathrm{cm}^{2}$ for chamber (2). The continuous straight line represents a track.

a value of about $98 \%$ for the correlation coefficient has been found. Centroid evaluation has been performed both in the $\mathrm{x}$ - (figure 7-bottom) and y-direction (not shown), and a value of about $5 \mathrm{~mm}$, similar to the nominal value of the spot size, has been measured. 

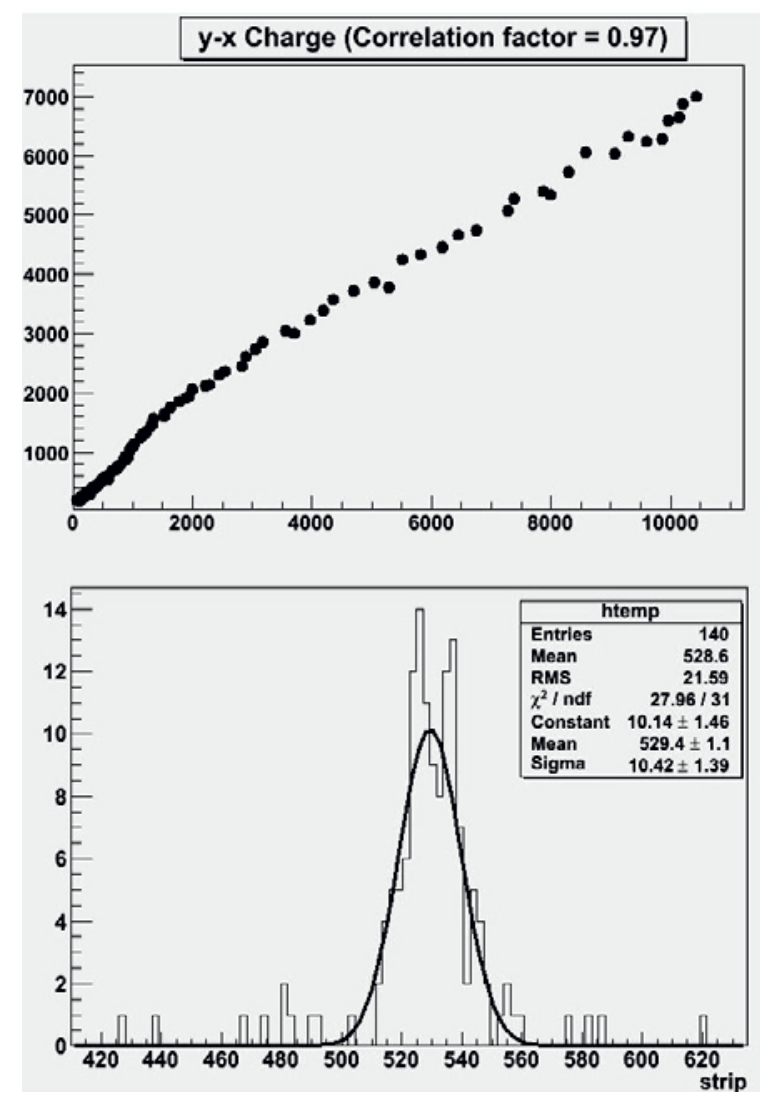

Figure 7. Charge correlation (top panel) and centroid in the $\mathrm{x}$ direction (bottom panel).

\section{Conclusions}

The main purpose of the test was to verify the overall functionality of the main solutions adopted in the first GEM modules under simple beam conditions. Both GEM hardware and readout electronics were under development and preliminary results on chamber efficiency and resolution were obtained. Summarizing, the GEM chambers operated fairly stably during the test and the results show reasonable indications about the detector performances. Further investigations about the test data are in progress.

\section{References}

[1] Thomas Jefferson National Accelerator Facility, www.jlab.org.

[2] The SBS project web site: http://hallaweb.jlab.org/12GeV/SuperBigBite/.

[3] $12 \mathrm{GeV}$ HallA upgrade web site: http://hallaweb.jlab.org/12GeV/.

[4] Sauli F., Nucl. Instr. Meth. A 386, 531 (1997).

[5] Bellini V. et al., JINST 7, C05013 (2012), proceedings of the MPGD 2012 conference.

[6] Ketzer B., Nucl. Instr. Meth. A 494, 142 (2002).

[7] Musico p. et al., 2011 IEEE Nuclear Science Symposium Conference Record.

[8] French M.J. et al., Nucl. Instrum. Meth. A 466, 359 (2002).

[9] $12 \mathrm{GeV}$ HallA upgrade web site: http://hallaweb.jlab.org/12GeV/.

[10] DESY Test Beams web site: http://adweb.desy.de/ testbeam/.

[11] Altunas C. et al., CERN-EP/2002-008, CERN, 21 January 2002. 